\title{
Motivational valence is determined by striatal melanocortin 4 receptors
}

\author{
Anna Mathia Klawonn, ${ }^{1}$ Michael Fritz, ${ }^{1}$ Anna Nilsson, ${ }^{1}$ Jordi Bonaventura, ${ }^{2}$ Kiseko Shionoya, ${ }^{1}$ Elahe Mirrasekhian, ${ }^{1}$ \\ Urban Karlsson, ${ }^{1}$ Maarit Jaarola, ${ }^{1}$ Björn Granseth, ${ }^{1}$ Anders Blomqvist, ${ }^{1}$ Michael Michaelides, ${ }^{2,3}$ and David Engblom ${ }^{1}$ \\ 'Department of Clinical and Experimental Medicine, Linköping University, Linköping, Sweden. Biobehavioral Imaging and Molecular Neuropsychopharmacology Unit, National Institute on Drug Abuse \\ Intramural Research Program, Baltimore, Maryland, USA. ${ }^{3}$ Department of Psychiatry, Johns Hopkins School of Medicine, Baltimore, Maryland, USA.
}

\begin{abstract}
It is critical for survival to assign positive or negative valence to salient stimuli in a correct manner. Accordingly, harmful stimuli and internal states characterized by perturbed homeostasis are accompanied by discomfort, unease, and aversion. Aversive signaling causes extensive suffering during chronic diseases, including inflammatory conditions, cancer, and depression. Here, we investigated the role of melanocortin 4 receptors (MC4Rs) in aversive processing using genetically modified mice and a behavioral test in which mice avoid an environment that they have learned to associate with aversive stimuli. In normal mice, robust aversions were induced by systemic inflammation, nausea, pain, and $\kappa$ opioid receptorinduced dysphoria. In sharp contrast, mice lacking MC4Rs displayed preference or indifference toward the aversive stimuli. The unusual flip from aversion to reward in mice lacking MC4Rs was dopamine dependent and associated with a change from decreased to increased activity of the dopamine system. The responses to aversive stimuli were normalized when MC4Rs were reexpressed on dopamine D1 receptor-expressing cells or in the striatum of mice otherwise lacking MC4Rs. Furthermore, activation of arcuate nucleus proopiomelanocortin neurons projecting to the ventral striatum increased the activity of striatal neurons in an MC4R-dependent manner and elicited aversion. Our findings demonstrate that melanocortin signaling through striatal MC4Rs is critical for assigning negative motivational valence to harmful stimuli.
\end{abstract}

\section{Introduction}

To promote survival, motivational circuits assign positive or negative valence to salient stimuli and the internal states they induce. When homeostasis is perturbed or severely challenged, such as during systemic inflammation, tissue damage, or intoxication, aversive circuit signaling induces discomfort, unease, and aversion (1-7). These affective responses might be beneficial during acute challenges, but they are often detrimental during chronic inflammatory and malignant diseases. Recent studies indicate that the molecules and circuits controlling energy balance also regulate the affective state (8). For example, Agouti-related protein (AGRP) neurons in the arcuate nucleus (Arc) and calcitonin gene-related peptide-expressing (CGRP-expressing) neurons in the parabrachial nucleus are important hubs in appetite control (9-11) and also elicit aversion upon activation $(4,5)$. The melanocortin system is a cornerstone of appetite and energy balance control (12). The appetite-reducing effect of melanocortin signaling is primarily mediated by proopiomelanocortin-expressing (POMC-expressing) cells in the Arc that activate melanocortin 4 receptors (MC4Rs) in the paraventricular hypothalamic nucleus $(12,13)$. Furthermore, MC4Rs in the ventral striatum have been

\section{Related Commentary: p. 2757}

Authorship note: AMK and MF contributed equally to this work. Conflict of interest: MM is a cofounder of and owns stock in Metis Laboratories Inc. Submitted: October 4, 2017; Accepted: May 1, 2018.

Reference information: / Clin Invest. 2018;128(7):3160-3170.

https://doi.org/10.1172/JCI97854 shown to mediate stress-induced anhedonia (14). However, the possible role of MC4Rs in other types of aversive processing has not been investigated.

\section{Results}

Mice lacking MC4Rs display indifference or preference to aversive stimuli. To determine whether MC4Rs are involved in aversive processing, we used a mouse line in which MC4R gene expression is blocked by a floxed STOP cassette. These mice develop obesity and other signs of MC4R deficiency $(13,15)$. We used conditioned place aversion (CPA) to characterize their response to aversive stimuli. In this paradigm, the mice are exposed to an aversive stimulus in a contextually specific manner and subsequently learn to avoid the associated environment. First, we tested to determine whether MC4Rs are important for aversion arising from systemic inflammation induced by i.p. injections of a low dose of bacterial cell wall lipopolysaccharide (LPS) $(10 \mu \mathrm{g} / \mathrm{kg})$. Normal WT mice learned to avoid the chamber where they received LPS. In contrast, mice lacking MC4Rs did not show aversion to the LPS-paired environment. Surprisingly, they instead showed a robust preference (Figure 1A), indicating that in these mice, LPS was a rewarding stimulus. To determine whether this unusual behavioral response was a general phenomenon or specific to LPS, we next examined whether mice lacking MC4Rs ( $M C 4 R-\mathrm{KO})$ reacted similarly to other aversive stimuli in the CPA paradigm. As expected, WT mice formed place aversions to i.p. injections of the $\kappa$ opioid receptor agonist U50488 (Figure 1B) and the nausea-inducing agent lithium chloride ( $\mathrm{LiCl}$ ) (Figure $1 \mathrm{C}$ ). In contrast, $M C 4 R-\mathrm{KO}$ mice reacted with preference toward these stimuli. When injected with formalin into the hind 
A
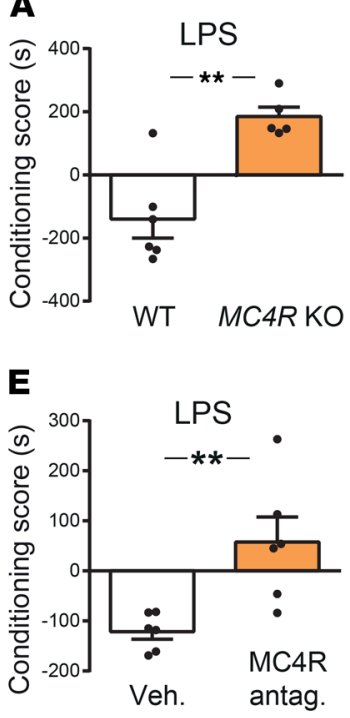

B

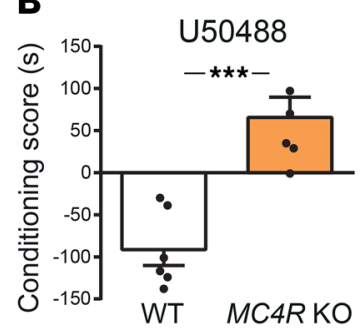

$\mathbf{F}$

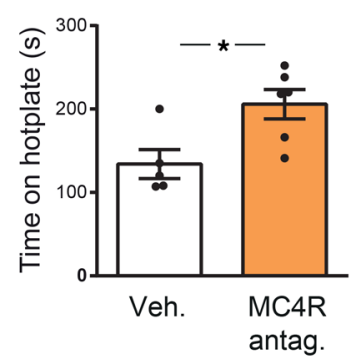

C

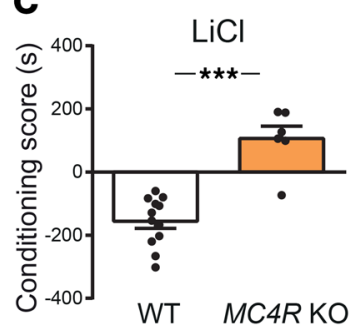

G



Figure 1. Mice lacking MC4 receptors display a preference for various aversive stimuli. (A-C) Mice with genetic deletion of MC4Rs (MC4R-KO) exhibited conditioned place preference in response to LPS ( $\mathbf{A}, n=6 \mathrm{WT} ; n=5 \mathrm{KO})$, the $\kappa$ receptor agonist U50488 (B, $n=6 \mathrm{WT} ; n=6 \mathrm{KO})$, and the nausea-inducing agent $\mathrm{LiCl}(\mathbf{C}, n=12 \mathrm{WT} ; n=6 \mathrm{KO}$ ). (D) The mutation also blocked aversion induced by inflammatory pain (formalin injection in the hind paw) ( $n=6 \mathrm{WT}$; $n=6 \mathrm{KO})$. (E) Pharmacological antagonism of MC4Rs (intranasal delivery of HS014) blocked the aversion induced by LPS in WT mice $\left(n=6 \mathrm{H}{ }_{2} \mathrm{O} ; n=6 \mathrm{HSO14}\right.$ ) and (F) decreased operant avoidance behavior toward a chamber with a hot floor compared with that of mice that had received $\mathrm{H}_{2} \mathrm{O}\left(n=6 \mathrm{H}_{2} \mathrm{O} ; n=6 \mathrm{HSO14}\right)$. Veh., vehicle; antag, antagonism. (C) MC4R antagonism also decreased nociceptive responses to formalin (lifting, shaking, biting, and licking of the affected paw) $\left(n=5 \mathrm{H}_{2} \mathrm{O} ; n=6 \mathrm{HSO14}\right)$. Results are displayed as mean $\pm \mathrm{SEM}$. ${ }^{*} P<0.05 ;{ }^{* *} P<0.01 ;{ }^{* * *} P<0.001$, Student's $t$ test (A-E and F); 2 -way ANOVA followed by Bonferroni's post hoc test (C).

paw, MC4R-KO mice formed neither an aversion nor a preference to the formalin-paired chamber (Figure 1D), indicating indifference toward pain. To investigate whether the loss of aversion to LPS was directly mediated by the lack of MC4Rs or caused by the resulting obesity or other developmental adaptations, we gave WT mice an MC4R antagonist (HSO14, intranasal administration) prior to LPS injection. The MC4R antagonist had an antiaversive effect that was similar to that caused by the genetic deletion (Figure 1E), demonstrating that neither developmental adaptations nor obesity were mediating the behavioral differences observed. Furthermore, the MC4R antagonist also affected the real-time avoidance of thermal pain. After administration of HSO14, mice given the choice between a chamber with a hot floor and a chamber with a neutral floor chose to spend a longer time on the hot floor compared with mice given vehicle (Figure $1 \mathrm{~F}$ ). This test involves an operant response and is not dependent on conditioning. Further, nociceptive responses to formalin paw pain were significantly attenuated by MC4R inhibition (Figure 1G).

In contrast to the absent or inverted aversive phenotypes, $M C 4 R-K O$ mice displayed normal LPS-induced anorexia (Figure 2A) and an almost intact LPS-induced hypothalamus-pituitaryadrenal axis activation (i.e., serum corticosterone elevation) (Figure 2B and Supplemental Figure 1A; supplemental material available online with this article; https://doi.org/10.1172/ JCI97854DS1). Furthermore, MC4R-KO mice exhibited LiClinduced hypothermia, which is a physiological correlate to nausea in rodents (16), in the same manner as WT mice (Figure 2C). These findings demonstrate that LPS and $\mathrm{LiCl}$ produce intact sickness symptoms in the MC4R-KO mice, but without eliciting the asso- ciated negative affective responses. Importantly, the inhibition or inversion of motivational behaviors was specific to aversive stimuli, as $M C 4 R-K O$ mice displayed normal conditioned place preference to cocaine (Figure 2D) and palatable food (Figure 2E). They were also able to acquire an operant task to obtain palatable food more effectively than WT littermates (Supplemental Figure 1B). These findings indicate normal processing of rewarding stimuli in MC4R-KO mice.

The dopamine response to LPS is inverted in MC4R-KO mice. The dopamine system is critical for many reward-related functions, but is also involved in aversive processing (17-21). We hypothesized that the abnormal response to aversive stimuli in $M C 4 R-K O$ mice coincided with altered striatal dopamine transmission. To examine this, we performed $\left[{ }^{11} \mathrm{C}\right]$ raclopride displacement studies using small animal positron emission tomography (PET). In the protocol used (Figure 3A), LPS injection and radioligand uptake in freely moving MC4R and WT mice was followed by PET. As expected, $\left[{ }^{11} \mathrm{C}\right]$ raclopride accumulated in the striatum (Figure $3 \mathrm{~A}$ ), illustrating proper uptake into the brain. We did not find differences between $M C 4 R-\mathrm{KO}$ and WT mice in dopamine D2 receptor binding using an autoradiography assay (Supplemental Figure $1 \mathrm{C})$, nor did $\left[{ }^{11} \mathrm{C}\right]$ raclopride uptake during baseline $(\mathrm{NaCl})$ conditions differ between the 2 genotypes (Supplemental Figure 1D). In line with our expectations, WT mice showed significantly higher $\left.{ }^{[11} \mathrm{C}\right]$ raclopride binding in the striatum and other extrastriatal D2 receptor-expressing regions after LPS than after $\mathrm{NaCl}$ (Figure 3, $\mathrm{B}$ and $\mathrm{C}$ ), illustrating decreased dopamine transmission, since dopamine readily displaces $\left[{ }^{11} \mathrm{C}\right]$ raclopride in vivo (22). As predicted by their behavior, there was no corresponding increase of 
A



B


D



$\mathbf{E}$



Figure 2. Nonmotivational responses to aversive stimuli and preference to rewarding stimuli are intact in $\mathbf{M C 4 R}-\mathbf{K O}$ mice. (A and B) Systemic inflammation led to a normal reduction in food intake (A, $n=6 \mathrm{WT} \mathrm{NaCl}$; $n=6 \mathrm{KO} \mathrm{NaCl} ; n=6$ WT LPS; $n=7 \mathrm{KO}$ LPS) and to a weakly attenuated corticosterone response (B, $n=5 \mathrm{WT} \mathrm{NaCl} ; n=5 \mathrm{KO} \mathrm{NaCl} ; n=7$ WT LPS; $n=8 \mathrm{KO}$ LPS) in mice lacking MC4Rs. (C) LiCl caused robust hypothermic responses in both WT and MC4R-KO mice ( $n=10 \mathrm{WT} \mathrm{NaCl} ; n=11 \mathrm{KO} \mathrm{NaCl}$; $n=10 \mathrm{WT} \mathrm{LiCl} ; n=11 \mathrm{KO} \mathrm{LiCl}$ ). (D and E) Mice lacking MC4Rs displayed normal conditioned place preference to $15 \mathrm{mg} / \mathrm{kg}$ cocaine i.p. as compared with WTs (D, $n=6 \mathrm{WT} ; n=9 \mathrm{KO})$ and had a slightly increased preference for palatable food (Nutella) (E, $n=12 \mathrm{WT} ; n=10 \mathrm{KO}$ ). Results are displayed as mean \pm SEM. ${ }^{*} P<0.05 ;{ }^{* *} P<0.01 ;{ }^{* * *} P<0.001$, Student's $t$ test ( $\mathbf{D}$ and E); ANOVA followed by Bonferroni's post hoc test (A and $\mathbf{B}$ ).

$\left[{ }^{11} \mathrm{C}\right]$ raclopride binding in $M C 4 R$-KO mice (Supplemental Figure $1 \mathrm{E})$. Instead, they displayed a significant decrease in $\left[{ }^{11} \mathrm{C}\right] \mathrm{raclo}-$ pride binding (equivalent to increased DA neurotransmission) after LPS injection (Figure 3, B and C). This decrease occurred specifically in the anterior part of the striatum between the nucleus accumbens (NAc) shell and the caudate putamen $(\mathrm{CPu}$ ) (Figure $3 \mathrm{C})$, an area that has been suggested as encoding hedonic value (23). These findings demonstrate that aversion coincided with decreases in striatal DA neurotransmission in normal mice, while MC4R-KO mice exhibited the exact opposite response, a flipped aversion-related striatal dopamine response that matched their flipped aversion-related behavioral phenotypes.

The abnormal response to aversive stimuli in MC4R-KO mice is dopamine dependent. To determine whether the LPS-induced increase in dopamine in $M C 4 R$-KO mice elicited their paradoxical aversion-related place preference, we examined whether inter- vention with dopamine signaling would normalize the aversive response of the $M C 4 R-\mathrm{KO}$ mice. Because striatal dopamine D1 receptors (D1R) are important for motivation and reward learning $(18,19)$, we injected a D1R antagonist (SCH23390; $0.2 \mathrm{mg} / \mathrm{kg})$ i.p. prior to administration of the aversive stimulus. Indeed, D1R antagonism blocked the development of LPS-induced place preference and reestablished the aversion in MC4R-KO mice (Figure 4A). D1R blockade also normalized the paradoxical place preference behavior we had observed after injection of the $\mathrm{\kappa}$ opioid receptor agonist (Figure 4B) and reestablished the aversion induced by formalin (Figure 4C). Our PET and D1R antagonist results indicate that $M C 4 R-\mathrm{KO}$ mice do not develop aversion, since they do not acquire the aversion-related drop in dopamine levels seen in normal mice during exposure to negative stimuli. Accordingly, we hypothesized that MC4R-KO mice would show normal aversion if their dopamine levels indeed were reduced, just as has been reported in WT mice (24). We tested this hypothesis using chemogenetic inhibition of ventral tegmental area (VTA) neurons via designer receptors exclusively activated by designer drug (DREADD) technology in MC4R-KO and WT mice. A viral vector (AAV8-hSyn-hM4Di-mCherry) expressing the inhibitory hM4Di DREADD (25) was injected into the VTA of mice (Figure 4, D and E). Chemogenetic inhibition of VTA neurons using clozapine $\mathrm{N}$-oxide (CNO) induced a normal place aversion in both WT and MC4R-KO mice (Figure $4 \mathrm{~F}$ ), indicating that $M C 4 R-\mathrm{KO}$ mice can form aversions if their dopamine transmission is inhibited. Collectively, these observations demonstrate that the transition from negative to positive motivational valence in the $M C 4 R$-KO mice is dependent on an inverted dopamine response acting on D1Rs.

MC4Rs in the ventral striatum are critical for aversion. MC4Rs are expressed on striatal neurons (26), including a population of D1R-expressing medium spiny neurons (MSNs) $(14,27)$. Using mice with reporter constructs for both MC4R and D1R, we confirmed that MC4Rs were expressed on D1R-expressing MSNs in both the dorsal (Figure 5A) and ventral striatum (Figure 5B). To test the involvement of MC4R-expressing D1R MSNs in aversive processing, we used the Cre-loxP system to selectively reactivate endogenous MC4R expression in D1R neurons in MC4R-KO mice. Reexpression of MC4Rs in D1R neurons, accomplished by removing the STOP cassette with an inducible Cre expressed under control of the D1R promoter, made the MC4R-KO mice capable of forming an aversive response to LPS (Figure 5C). In contrast, reactivation of MC4R expression in dopamine cells, using a DATCre-ER mouse line, had no effect (Figure 5D). In accordance with the normalized reaction to LPS, reexpression of MC4Rs in D1R neurons in MC4R-KO mice was sufficient to induce aversive behaviors in response to the $\kappa$ receptor agonist U50488 (Figure 5E) and formalin-induced pain (Figure 5F). To determine whether this effect was specific to striatal MC4Rs, we used stereotaxic injection of viral vectors expressing Cre to selectively rescue MC4Rs in the striatum of $M C 4 R-\mathrm{KO}$ mice. As expected, mice with striatal reexpression of MC4Rs displayed normal aversion to LPS (Figure 5, G and H). Collectively, these findings indicate that MC4Rs on D1R-expressing striatal MSNs are critical regulators of aversive signaling.

Striatum-projecting POMC neurons induce aversion. Striatal MC4Rs are targeted by a population of POMC neurons arising from the Arc (14). Accordingly, we hypothesized that activation of 
A

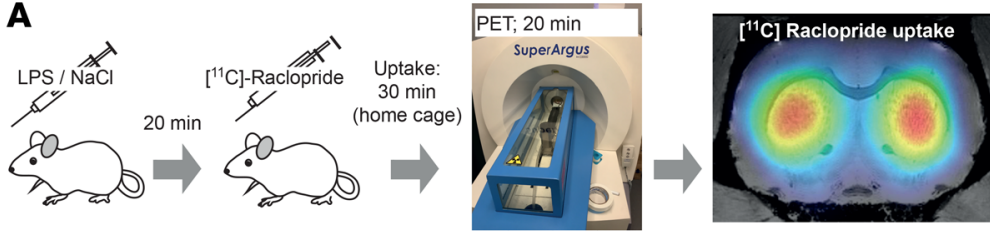

B

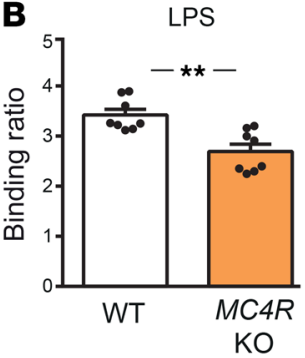

C



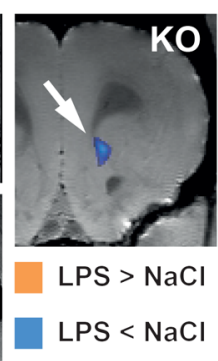

Figure 3. MC4R-KO mice display an inverted dopamine response to inflammation. (A) Timeline for the small-animal PET imaging experiment with MC4R-KO and WT animals treated with LPS or $\mathrm{NaCl}$ and ["1 $\mathrm{C}$ ]raclopride injections ( $n=4 \mathrm{WT} ; n=4$ $\mathrm{KO})$ together with an example of $\left.{ }^{[1} \mathrm{C}\right]$ raclopride uptake. (B and $\mathbf{C}$ ) WT animals had increased $[" C]$ raclopride binding (orange labeling in C) in the dorsal and ventral striatum, lateral septum, and ventral pallidum after LPS administration. No such increase was seen in MC4R-KO mice. ${ }^{*} P<0.01$. (B and $\mathbf{C}$ ) The MC4R-KO mice instead displayed decreased $\left[{ }^{11} \mathrm{C}\right]$ raclopride binding (blue labeling, $P<0.001)$ in a border area between the ventral and dorsal striatum. The labeling is superimposed onto an MRI template. Results in $\mathbf{B}$ are displayed as mean \pm SEM and are based on analysis of both sides. Blue or orange labeling indicates changes identified as significant with Voxel statistics linear model analyses (ANOVA) in statistical parametric mapping (SPM) with post hoc $t$ test; clusters of more than 500 voxels, $P<0.001$. VP, ventral pallidum. arcuate POMC neurons would be sufficient to cause aversion. We expressed the stimulatory hM3Dq DREADD in POMC cells of the Arc by injecting Cre-dependent viral vectors (AAV8-DIO-hM3DqmCherry) into POMC-Cre mice (Figure 6, A and B). Indeed, activation of POMC cells by systemic administration of $\mathrm{CNO}$ induced a robust CPA (Figure 6C). This response was not seen in a control group also injected with $\mathrm{CNO}$, ruling out the possibility that the response was driven by off-target effects of CNO (28). Next, we selectively activated arcuate POMC neuron projections to the ventral striatum by exploiting the retrograde transport of the AAV8 serotype (Figure 6D). Injection of the vector (AAV8-DIO-hM3DqmCherry) in the ventral striatum of POMC-Cre mice led to expression of hM3Dq in a subpopulation of POMC neurons in the Arc (Figure 6E) and selective activation of this neuronal population, by $\mathrm{CNO}$ administration, induced a robust aversion (Figure $6 \mathrm{~F}$ ).

POMC neurons activate the ventral striatum in a MC4R-dependent manner. To investigate the connectivity between MC4R-expressing striatal neurons and POMC terminals projecting to this region, we used optogenetic stimulation in slice recordings. To this end, we crossed POMC-Cre mice with a mouse line expressing Creinducible channelrhodopsin-2 (Chr2), resulting in mice with selective expression of Chr2 in POMC neurons. Cell-attached recordings revealed that optogenetic activation $(20 \mathrm{~Hz}, 1$ minute) of striatal POMC terminals increased the firing frequency in around one-fourth of striatal neurons (Figure 7A). This is consistent with the finding that a subpopulation of striatal neurons expresses MC4Rs (Figure 5, A and B). We found that blockade of glutamatergic signaling by DNQX moderately decreased the activity of these neurons. Furthermore, addition of the MC4R antagonist HSO14 blocked the activity (Figure 7A). These data indicate that arcuate POMC neurons project to MC4R-expressing neurons in the striatum, where POMC activation increases the firing frequency of these neurons via activation of MC4Rs. This is consistent with previous work identifying a G-protein-independent mechanism by which MC4Rs cause burst firing (29). In order to demonstrate this connectivity pattern in vivo, we used DREADD-assisted metabolic mapping (DREAMM) (30) and examined how activation of POMC neurons projecting to the ventral striatum affected brain activity patterns in vivo in freely moving mice. Mice expressing hM3Dq in POMC neurons were injected i.p. with either vehicle or CNO followed by approximately $500 \mu \mathrm{Ci}$ of $\left[{ }^{18} \mathrm{~F}\right]$ fluorodeoxyglucose (FDG) and placed back into their home cage for 30 minutes, during which time FDG accumulated and was trapped in metabolically active cells, thereby serving as a marker of cell activity. Subsequently, mice were quickly anesthetized and scanned in a small animal PET scanner (Figure 7B). We found that hM3Dq activation of POMC neurons led to significant increases in brain FDG uptake, with the greatest effect localized in the ipsilateral ventral part of the dorsal striatum (Figure 7, C-E). Furthermore, increased contralateral FDG uptake occurred in both the ventral and dorsal striatum and the posterior insular cortex (PI). These structures are involved in the processing of affective states, including aversion $(3,17,18,31)$. DREAMM responses were exclusively excitatory (increased metabolic activity). The upregulation of ipsilateral striatal metabolic activity is in accordance with our above finding that optogenetic POMC neuron stimulation increased striatal neuron activity in an MC4R-dependent manner. Collectively, these findings indicate that arcuate POMC neurons activate MC4Rexpressing neurons in the striatum to drive aversive signaling and aversion-related behaviors.

\section{Discussion}

We identify striatal MC4Rs as critical regulators of aversive processing. Our findings strongly suggest that MC4Rs induce aversion by inhibiting dopamine signaling. Intriguingly, the aversions to some stimuli were even flipped into preferences in the mutant mice. In response to LPS, we consistently saw preferences in $M C 4 R-K O$ mice. Pooled analysis of all naive MC4R-KO mice given LPS demonstrated a preference score that was highly significantly different from 0 ( $P=0.0005$; column statistics, 1-sample $t$ test), strongly suggesting a real preference and not only a lack of aversion. In accordance with this observation, our imaging data show that MC4R deletion not only eliminated the LPS-induced reduction in dopamine, but also led to an increase in dopamine in the striatum. However, in response to aversive stimuli, the flip to preference was generally less strong than the loss of aversion in the $M C 4 R-K O$ mice, and the increase in dopamine signaling upon LPS was less extensive than the drop seen in WT mice. These findings suggest that, whereas the flip from aversion to preference toward aversive stimuli upon removal of MC4Rs is conceptually highly interesting, the lack of aversion and lack of dopamine decrease in 
A
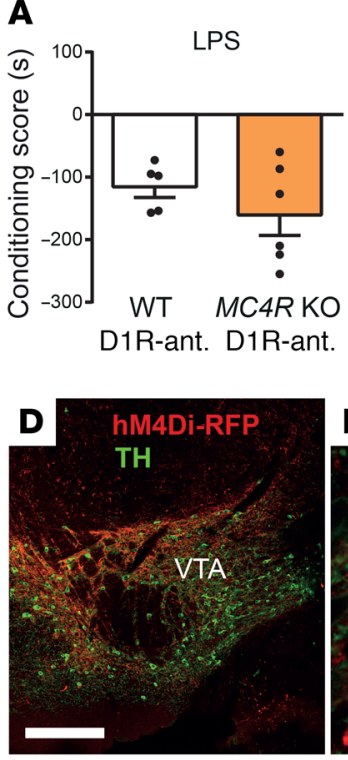

B

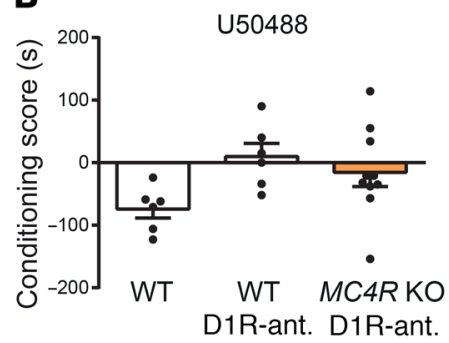

C

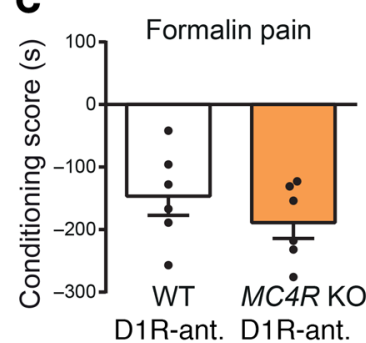

Figure 4. The preference for aversive stimuli in MC4R-KO mice is mediated by dopamine. (A-C) When a D1R antagonist, SCH23390, was administered prior to the aversive stimuli, MC4R-KO mice and WT mice responded similarly to LPS (A, $n=5 \mathrm{WT} ; n=6 \mathrm{KO}$ ), U50488 (B, $n=6$ WT NaCl; $n=6$ WT SCH23390; $n=10 \mathrm{KO}$ SCH23390), and formalin-induced pain (C, $n=6$ WT; $n=6$ KO). (D and E) Representative example (out of $n=10$ ) of hM4Di-RFP expression in the midbrain of mice injected with a viral vector expressing the inhibitory DREADD hM4Di. Arrows indicate examples of dual-labeled cells. (F) Chemogenetic inhibition of neurons in the region of the VTA induced normal aversive responses in both WT and MC4R-KO mice $(n=5 \mathrm{WT} ; n=5 \mathrm{KO})$. Results are displayed as mean \pm SEM. Statistical significance was assessed by Student's $t$ test (A, C, and $\mathbf{F})$ and ANOVA (B). Scale bars: $250 \mu \mathrm{m}$ (D); $25 \mu \mathrm{m}$ (E).

response to aversive stimuli are more general and robust consequences of MC4R deletion or inhibition. To learn more about the reinforcing properties of LPS and other aversive stimuli in MC4R$\mathrm{KO}$ mice, it would be interesting to determine whether $M C 4 R-\mathrm{KO}$ mice would self-administer these substances. However, LPS, LiCl, and $\kappa$ receptor antagonists all have slow mechanisms of action that make operant training unlikely to succeed.

While we demonstrate that MC4Rs in the striatum are critical for aversive processing, previous studies show that the appetiteregulating effect of melanocortins is primarily mediated by MC4Rs in the paraventricular hypothalamus (13). Only a very minor effect on body weight and basal food intake was seen when MC4Rs were reexpressed on D1R neurons of MC4R-KO mice (32). MC4Rs have been suggested as being involved in inflammation-induced appetite loss (33), but we found an intact LPS-induced anorexia in the MC4R$\mathrm{KO}$ mice. The intact anorexia in combination with the blocked aversion supports previous findings suggesting that the affective component and the anorexic component of systemic inflammation are mediated by different mechanisms and circuits (7). It could be suggested that the loss of aversion and the abnormal preferences seen in $M C 4 R$-KO mice might be indirect effects related to their obesity and increased appetite. For example, LPS-mediated relief of hunger in the mutant mice could be rewarding and thus compensate for the aversive properties of the LPS-induced affective state. However, such explanations are very unlikely, since we also see a loss of aversion in response to formalin and a $\kappa$ receptor agonist, which are not strongly appetite reducing. Further, in the experiments using MC4R inhibitors, the mice did not experience any hunger related to MC4R inhibition under basal conditions or in the saline-paired chamber. Finally, mice with rescue of MC4Rs in D1R neurons displayed normal aversions despite being almost as obese as $M C 4 R$ KO mice $(27,32)$.

Our findings demonstrate that POMC neurons projecting to the ventral striatum induce aversion by activation of MC4Rs. In contrast, most other projections to the ventral striatum previously investigated signal reward. Thus, activation of input from the ven- tral hippocampus, the basolateral amygdala, and the prefrontal cortex all induce place preference $(34,35)$. Similar to the POMC projection, glutamatergic input from the VTA and the periventricular thalamic nucleus both induce aversion $(36,37)$, but they do so by targeting parvalbumin-positive GABAergic interneurons (36) and D2R-expressing MSNs (37), respectively. In contrast, our findings indicate that POMC neurons signal aversion by activation of a subpopulation of D1R neurons. Consistently, stress-induced anhedonia has been reported to be mediated by MC4R-dependent synaptic plasticity in D1R MSNs in the ventral striatum (14).

Pathways previously found to mediate aversion to specific negative stimuli are highly diverse (1, 2, 6, 7, 37-40). In contrast, here we show that MC4Rs, localized on striatal D1R MSNs, control behavioral responses to a variety of aversive stimuli and thereby play a more generalized role in aversive processing. Our findings indicate that MC4Rs assign negative valence to salient stimuli, effectively acting as gatekeepers whose role is to hinder aversive signals from inducing reward. Such a mechanism has obvious adaptive value for survival, but in the chronically ill, it is an unwanted cause of suffering. The very strong and broad antiaversive effect of MC4R inhibition makes it an interesting strategy for reducing malaise and depressive symptoms associated with chronic diseases, including inflammatory conditions and terminal states.

\section{Methods}

Animals. All experiments were done using naive mice. The animals were single housed for a minimum of 48 hours prior to the experiments and kept in a pathogen-free facility on a regular 12-hour light/12-hour dark cycle. For behavioral, PET, and electrophysiology experiments, only male mice were used, whereas both sexes were used in biochemical and food-intake experiments. Each experimental group consisted of mice from at least 2 litters. All mice were more than 6 weeks old at the onset of experiments, and the typical age was 8 to 20 weeks. Food and water were provided ad libitum, and all experiments were performed during the light phase, unless otherwise noted. 



Figure 5. MC4Rs on striatal neurons expressing the D1R are critical for aversion. (A and B) Micrographs from the striatum (A, CPu; B, NAc) showing MC4R expression (eGFP) in green and D1R expression (tdTomato) in red. (C) MC4R-KO mice with rescue of MC4R expression selectively in D1R-expressing cells responded with place aversion to LPS ( $n=5 \mathrm{WT} ; n=11 \mathrm{KO} ; n=7$ MC4R-D1-rescue). resc, rescue. (D) Rescue of MC4Rs selectively on dopamine cells did not restore aversion ( $n=5 \mathrm{WT} ; n=4 \mathrm{KO} ; n=6 \mathrm{MC} 4 \mathrm{R}$-DAT-rescue). (E and $\mathbf{F}$ ) Rescue of MC4Rs on D1R cells normalized the aversive responses of MC4R-KO mice to the $\mathrm{k}$ receptor agonist U50488 (E, $n=8 \mathrm{WT} ; n=10 \mathrm{KO} ; n=7 \mathrm{MC}$ RR-D1-rescue) and to inflammatory pain (F, $n=7 \mathrm{WT} ; n=8 \mathrm{KO} ; n=6 \mathrm{MC} 4 \mathrm{R}$-D1-rescue) (F). (G) Expression of Cre in the striatum was induced by a local injection of a viral vector together with a Cre-dependent eGFP-expressing viral vector. LV, lateral ventricle. (H) MC4R-KO mice with rescue of MC4R expression in the striatum displayed intact aversive responses to LPS ( $n=6$ WT AAV5-Cre; $n=9$ KO AAV5-GFP; $n=5$ MC4R-rescue in KO with AAV5-Cre). Results are displayed as mean \pm SEM. ${ }^{*} P<0.05 ;{ }^{* *} P<0.01$, ANOVA followed by Bonferroni's post hoc test. Scale bar: $20 \mu \mathrm{m}$.

MC4R-STOP-flox and Chr2-STOP-flox, POMC-Cre, Drd1 Cre-ER, and Dat Cre-ER mice have been previously described in the literature (13, 41-44). The Drd1 Cre-ER line was provided by Jan Rodriguez Parkitna (Institute of Pharmacology, Polish Academy of Sciences, Krakow, Poland). MC4R-STOP-flox, MC4R-eGFP, Drd1-tdTomato, Chr2-flox, POMC-Cre, and WT C57BL/6J mice were purchased from The Jackson Laboratory. All mice had a C57BL/6 background, with the exception of POMC-Cre (mixed FVB/N and C57BL/6J) and Chr2-STOP-flox (mixed C57BL/6 and 129). Cre-recombinase activity was induced by administering tamoxifen dissolved in sunflower seed oil/alcohol mixture at a 10:1 ratio. The mixture was i.p. injected at $1 \mathrm{mg}$ per mouse twice a day for 5 days.

Blinding and randomization. All conditioning experiments were under blinded conditions unless otherwise stated in the text describing the specific experimental procedure. Methods using automatized data collection, i.e., telemetric temperature recordings, photometrybased corticosteroid assays, PET scans, electrophysiology recordings, etc., were not under blinded conditions. Animals were randomized for treatment throughout the study and were selected to ensure mixed balanced groups during conditioning sessions.

Drugs and injections. LPS from E. coli (055:B5, Sigma-Aldrich) was administered i.p. at a concentration of $10 \mu \mathrm{g} / \mathrm{kg} 10$ minutes prior to CPA training or 1 hour before food-intake measurements. U50.488 (Tocris Bioscience) and $\mathrm{LiCl}$ (Sigma-Aldrich) were injected i.p. at $2.5 \mathrm{mg} / \mathrm{kg}$ and $130 \mathrm{mg} / \mathrm{kg}$, respectively, directly before CPA training. Diluted formalin $(2.5 \% ; 20 \mu \mathrm{l})$ (Sigma-Aldrich) was injected below the skin on the dorsal side of the hind paw to induce acute inflammatory pain for CPA. HSO14 (Tocris Bioscience) at $50 \mu \mathrm{g} / 5 \mu \mathrm{l}$ was dissolved in Milli-Qwater (MilliporeSigma) and administered in each naris 30 minute prior to LPS
CPA training, operant thermal place aversion, or nociceptive responding to formalin. SCH23390 (Tocris Bioscience) was given i.p. (0.2 mg/ kg) 15 minutes prior to CPA training. CNO from Enzo was injected i.p. at $2 \mathrm{mg} / \mathrm{kg} 10$ minutes before CPA training. All compounds were diluted in physiological saline $(0.9 \% \mathrm{NaCl})$ unless otherwise stated, and i.p. injections were in a volume of $100 \mu \mathrm{l} / 25 \mathrm{~g}$ mouse.

Intranasal infusions. Mice were briefly anesthetized with isoflurane before receiving $50 \mu \mathrm{g}$ HSO14/5 $\mu \mathrm{l}$ Milli-Q water or just Milli-Q water per naris. Intranasal infusions were done according to a previously published protocol for delivery of drugs to the brain via the olfactory, rostral migratory stream, and trigeminal routes (45). A micropipette was used for delivering a droplet to each naris while the mouse was held in an upright position. When the drops had been inhaled, the mouse was gently tilted backwards to avoid the liquid running back into the pharynx. Following the infusion, the mouse was left on its back in its home cage before placement in the behavioral arena.

Virus generation. The viral vectors used for Cre-induced rescue of MC4Rs were made as previously described (7). AAV8-hSyn-hM4D(Gi)-mCherry for midbrain neuronal inhibition and AAV8-hSynDIO-hM3D(Gq)-mCherry for POMC-Cre-dependent chemogenetic activation of neurons were obtained from the Vector Core at the University of North Carolina (Chapel Hill, North Carolina, USA).

Stereotaxic surgeries. For all stereotaxic surgeries, mice were anesthetized with $5 \%$ isoflurane induction, placed in the stereotaxic frame (Leica Biosystems), and maintained at 1.0\%-1.5\% isoflurane during surgery. Adeno associated virus (AAV) vectors were bilaterally injected at a rate of $100 \mathrm{nl} / \mathrm{min}$ into either the midbrain, striatum, Arc, or NAc shell, using a gastight Hamilton Neuros syringe (33G). Midbrain injections of Gi-DREADD-expressing virus (250 nl per injection site) 
A
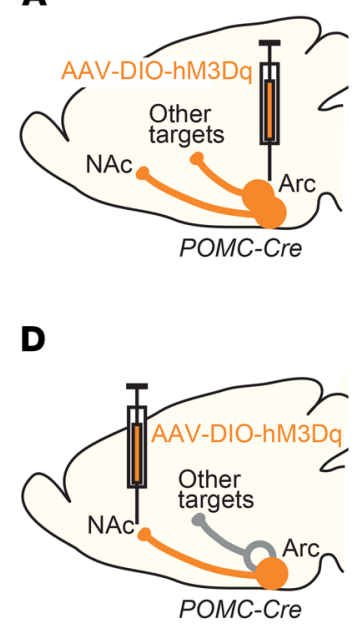
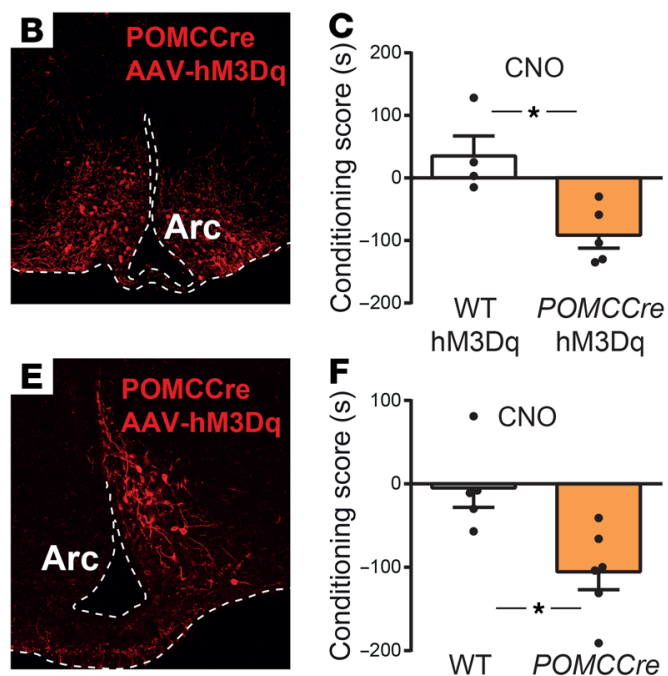

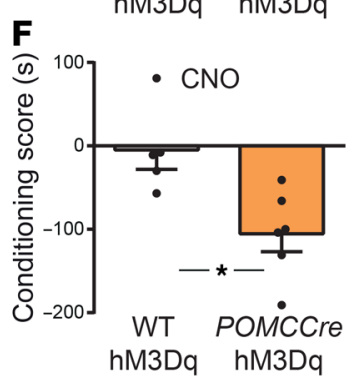

Figure 6. Activation of POMC neurons projecting to the ventral striatum is aversive. ( $A$ and $\mathbf{B}$ ) Expression of hM3Dq-RFP in POMC cells of the Arc after injection of a Cre-dependent viral vector in POMC-Cre mice (representative example from $n=5$ ). (c) Chemogenetic activation of arcuate POMC cells by i.p. injection of CNO-induced CPA ( $n=4$ WT; $n=5$ POMC-Cre). (D and E) Expression of hM3Dq-RFP in POMC cells of the Arc after striatal injection of Cre-dependent viral vectors with the potential to be retrogradely transported in POMC-Cre mice (representative example from $n=6)$. (F) Chemogenetic activation of arcuate POMC neurons projecting to the striatum-induced aversion ( $n=5 \mathrm{WT} ; n=6$ POMC-Cre). Results are displayed as mean \pm SEM. ${ }^{*} P<0.05$, Student's $t$ test. were done with stereotaxic coordinates: (anterior/posterior [AP], -3.4; medial/lateral [ML], \pm 0.48 ; dorsal/ventral [DV], -4.6). AAV5 (250 nl per injection site) for Cre-dependent expression was delivered in the striatum (AP, $+1.1 ; \mathrm{ML}, \pm 1.2 ; \mathrm{DV},-3.0$ ). For direct activation of POMC neurons, Gq-DREADD-expressing virus was delivered (250 $\mathrm{nl}$ per injection site) into the Arc (AP, -1.4; ML, \pm 0.3 ; DV, -5.8). Retrograde activation of POMC neurons using DREADD-Gq (600 nl per injection site) was done by injections in the NAc shell (AP, +1.1; ML, \pm 0.5 ; DV, -4.5). The injection needle was left in place for 10 minutes after injection to ensure proper diffusion. Animals received analgesic treatment during the first 48 hours after surgery with buprenorphine $(25 \mathrm{mg} / \mathrm{kg}$; Temgesic, RB Pharmaceuticals). Behavioral experiments or PET imaging was conducted 3 to 5 weeks after the viral injections. Following the behavioral experiments, construct-expression and injection-site location were validated with fluorescent immunohistochemistry. Only results from animals with correct injection placement and expression were included in the manuscript. The hM4DGi-VTA inhibition experiment was replicated once without any subject being excluded. In the Cre virus rescue experiments, 2 animals were excluded due to engagement of the ventricle or low viral expression. Experiments with Cre virus were replicated twice. For the hM3DGq-chemogenetic activation experiments, expression in 1 hemisphere was sufficient for stable behavior. Three animals were excluded from the DREAMM experiment due to lack of expression.

CPA and preference. We used a balanced place conditioning procedure to measure aversion and preference, applying a 3-chambered Panlab Spatial Place Preference Box (Harvard Apparatus) as described previously (7). All training and test sessions were conducted during the light phase of the 12-hour light/12-hour dark cycle. On day 1, during a 15-minute pretest, the individual mouse was allowed to move freely between the chambers of the box. Time spent in each compartment was manually recorded by independent experimenters blinded to genotype and/or treatment, with an exception for the initial experiments in which the MC4R-KO mice were visually distinguishable from the WT mice due to their obese phenotype. To ensure explorative behavior during the pretest, each mouse had to cross the corridor, entering the opposing chamber a minimum of 5 times to be included in the experiment. Any animals that spent more than $66 \%$ of their time during pretests in either of the conditioning chambers were discarded from the study. Mice were assigned to vehicle- or stimulus-paired compartments in a manner that avoided reinforcing natural bias, e.g., LPS injections were paired with the most preferred chamber identified during pretest and cocaine with the least preferred chamber. This method has been shown to produce reliable conditioned place responses comparable to those resulting from other paradigms (46). On day 2 of place aversion conditioning, mice were injected with aversive stimuli (LPS, U50.488, LiCl) or CNO before confinement for 1 hour in one of the compartments. The following day, the mice were injected with saline in the opposite chamber. This alternating training procedure was continued for 8 consecutive days, until day 10 , when the CPA was assessed by allowing the mice to freely explore all compartments of the box for 15 minutes. The conditioning score was calculated by subtracting the time the mouse spent in the stimuluspaired chamber during the pretest from that spent in the same chamber during the posttest. For the experiments on formalin-induced pain aversion, the training protocol was slightly modified. Here, the training spanned 2 consecutive days, with 2 sessions each day. In the morning session, mice were briefly anesthetized with isoflurane and confined to the nonpreferred chamber for 60 minutes. In the afternoon, mice were anesthetized with isoflurane, injected with diluted formalin in the dorsal part of the back paw, and confined to the conditioning chamber for 60 minutes. Place preference experiments using cocaine or palatable food were conducted using the same test protocol as that used for place aversion, but these employed an adjusted training schedule. In the mornings, mice were given a nonreinforcing control (saline i.p. or an empty plate) in the vehicle chamber, and in the afternoon, 4 hours later, mice were given a positive reinforcer (cocaine $15 \mathrm{mg} / \mathrm{kg}$ i.p. or Nutella [Ferrero] on a small plate) in the conditioning chamber. Each training session lasted 15 minutes and was repeated over 4 consecutive days.

Operant thermal place aversion. For the operant responding to thermal discomfort, a 2-chamber arena with temperature-regulated floors and infrared beam detection (series 8, IITC Life Sciences) was used. The chambers had distinct patterns (checkered or striped) on the walls. The floor temperature of 1 chamber was set to $30 \pm 0.1^{\circ} \mathrm{C}$ and the other was $45 \pm 0.1^{\circ} \mathrm{C}$. Thirty minutes prior to the operant test, mice 
A
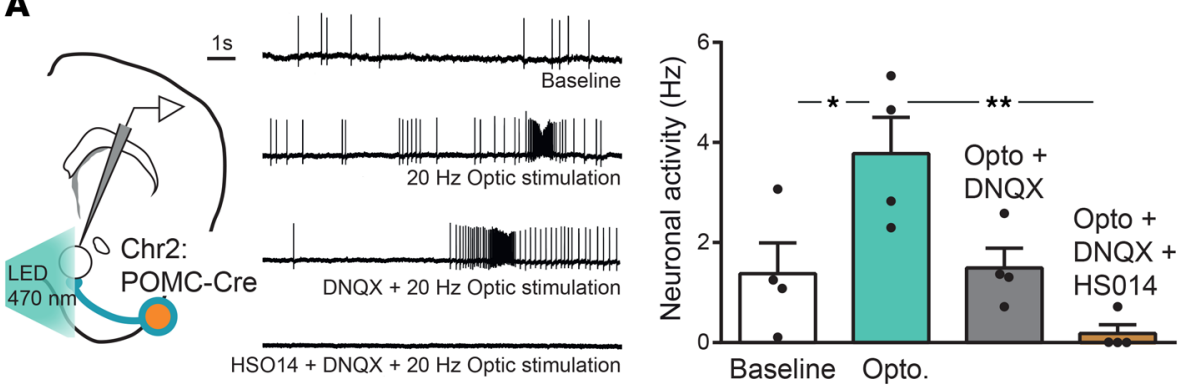

B
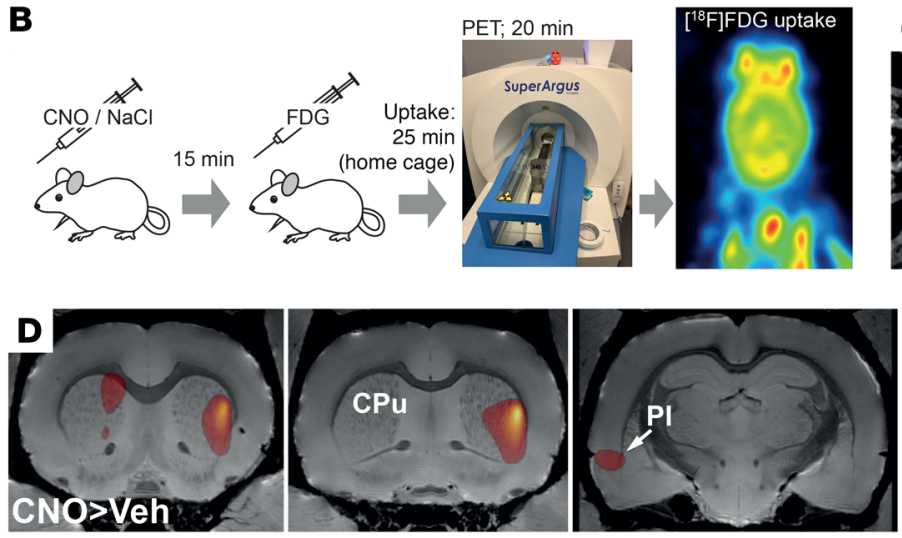
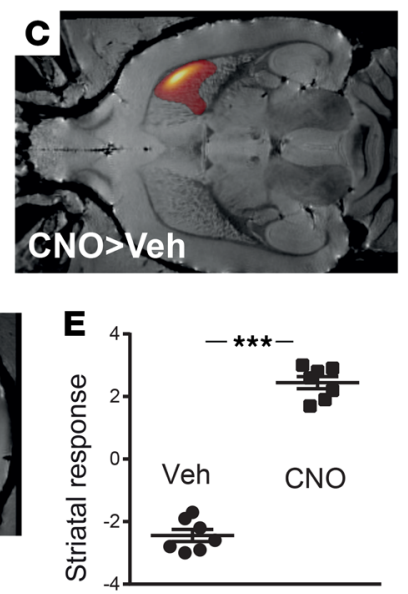

Figure 7. POMC neurons projecting to the ventral striatum activate striatal neurons in a MC4R-dependent manner.

(A) Cell-attached recordings from the striatum showed that optogenetic activation of POMC projection neurons increased cell firing in a glutamate receptor- and MC4R-dependent manner $(n=4$ out of 18 Chr2: POMCCre). Representative traces ( 15 seconds) from the end of a recording, where most action potentials occurred. (B) Timeline of the experiment using DREAMM and an image of $\left[{ }^{18} \mathrm{~F}\right]$ FDC uptake in the brain. (C-E) DREAMM using $\left[{ }^{18} \mathrm{~F}\right] \mathrm{FDC} \mathrm{PET}$ after $\mathrm{CNO}$-induced activation of POMC cells projecting to the ventral striatum. hM3Dq was expressed in the same way as in Figure 6D. Clear activation (orange labeling; $P<0.01$ ) was seen in the striatum on the same side as the viral injection ( $n=7$ POMCCre) as well as in both ventral and dorsal striatum and the PI of the contralateral side. Voxel statistics analysis in MATLAB and SPM; clusters of more than 100 voxels,

$P<0.01$ (C and $\mathbf{D})$. Results are displayed as mean \pm SEM. ${ }^{*} P<0.05 ;{ }^{* *} P<0.01$;

${ }^{* *} P<0.001$, ANOVA followed by Bonferroni's post hoc test (A); Student's $t$ test (E). received intranasal injections of either $\mathrm{H}_{2} \mathrm{O}$ or HSO14 as described under Intranasal infusions. The animals were then allowed to freely choose between the 2 chambers during a 15-minute test, while their entries and time spent in each chamber were automatically recorded.

Nociceptive responding to formalin. Mice were kept in a transparent Plexiglas box $(20 \times 15 \times 25 \mathrm{~cm})$ for 30 minutes of acclimatization. The mice were then briefly anesthetized with isoflurane prior to a formalin injection in the right hind paw and subsequently received intranasal infusions of either $\mathrm{H}_{2} \mathrm{O}$ or HSO14. The animals were placed back in the Plexiglas box and allowed to recover from anesthesia for around 5 minutes. Their behavior was videotaped for 60 minutes with a Canon LEGRIA HF R48 camera. Nociceptive behaviors, such as paw lifting (category I) and licking, biting, and shaking time (category II), were measured by experimenters offline, and nociceptive scores were calculated for 5-minute intervals, as follows: Nociception score (N.S.) = ((time of paw lifting) $\times 1+$ (time of paw shaking, biting, and licking $) \times$ 2)/300 (6).

Operant runway. The operant runway experiment was conducted in the light phase of the 12-hour light/12-hour dark cycle, using a custom-made mouse runway built by AgnTho's A/B. The runway was equipped with a white start chamber $(14 \times 14 \times 25 \mathrm{~cm})$ that had a retractable door and a gray corridor $(80 \mathrm{~cm}$ long). At the end of the corridor, there was a goal chamber (same size as the start chamber) equipped with a retractable door and distinct visual cues: black on white dotted wallpaper, a black floor, and a cue light. Mice were habituated to Nutella on a tile in their home cages 2 days before and food restricted for 4 hours prior to the experiment. On the day of the experiment, at the start of each run, a small amount of Nutella was placed in the goal chamber and the door was left open to allow free access. Each mouse was recorded in 5 consecutive trials (runs) separated by 1 hour, during which time the mouse was placed back in its home cage. The time that an animal needs to obtain the reward (runtime) is considered to be inversely proportional to the strength of that specific stimulus (47). Each run was initiated after the mouse was carefully placed into the start chamber by opening the door to the corridor. The mice were allowed to move freely between the chambers of the runway during the test time, and mice that didn't reach the goal chamber within 90 seconds were gently guided there by the experimenter. Time spent until the mouse reached the goal chamber was manually recorded by more than one independent experimenter. After arriving in the goal chamber, the mouse was confined there for 2 minutes by carefully closing the door behind it, and the cue light was turned on. Each individual runtime included the time the mouse spent in the start chamber after the door was opened and the time the mouse needed to reach the goal chamber. All chambers of the runway were carefully cleaned with isopropanol between runs.

Food intake. Before the experiments, mice were single-housed for at least 7 days. One hour before dark-period onset, food was withdrawn and LPS or saline was injected i.p. At lights out, mice were given free access to a preweighed amount of standard chow, and food intake was measured 6 hours after refeeding.

Telemetric temperature recordings. Core body temperature was monitored using continuous telemetry with an abdominal transmitter. Mice were briefly anesthetized with $5 \%$ isoflurane induction and maintained at $1.0 \%-1.5 \%$ isoflurane during i.p. implantation of transmitters. Telemetric transmitters for recording core body temperature were obtained from Data Science International. Animals received analgesic treatment prior to surgery and during the first 48 hours after surgery with buprenorphine $(25 \mathrm{mg} / \mathrm{kg}$; Temgesic, RB Pharmaceuticals) and were allowed to recover for at least 5 days before the experi- 
ment. From the surgery to the end of the experiment, mice were kept in a thermoneutral environment $\left(29^{\circ} \mathrm{C}\right)$. Baseline temperature was recorded for 72 hours prior to the experiment to ensure normal body temperature with circadian variation. On the day of the experiment, $\mathrm{LiCl}$ or saline was injected i.p. and the body core temperature was recorded for at least 6 hours.

Corticosterone assay. Corticosterone levels were determined from the heart blood of MC4R-KO mice and their WT littermates following LPS or saline i.p. injections. Animals were euthanized by asphyxiation with $\mathrm{CO}_{2}$, and blood was taken from the hearts via a thoracotomy through the diaphragm using a syringe with a 23-gauge needle. Blood samples were collected 2 or 3.5 hours after injections. Blood samples were stored at $4^{\circ} \mathrm{C}$ overnight before being centrifuged at 2,650 rcf/g for 20 minutes. The corticosterone content was determined using a Corticosterone EIA Kit (Enzo) according to the manufacturer's protocol.

Fluorescent immunohistochemistry. Animals were euthanized with $\mathrm{CO}_{2}$ asphyxiation, and brains were collected after intracardial perfusion with saline and 4\% PFA in PBS ( $\mathrm{pH}$ 7.4). The brains were postfixed for 4 hours in $4 \%$ PFA and subsequently cryoprotected in a $30 \%$ sucrose PBS solution overnight. Coronal sections $(40 \mu \mathrm{m})$ were cut on freezing microtome, collected in cold cryoprotectant buffer (0.1 M phosphate buffer, 30\% ethylene glycol, 20\% glycerol), and stored at $-20^{\circ} \mathrm{C}$ until further use. For immunofluorescent labeling, free-floating sections were washed in PBS, incubated in blocking solution (1\% BSA and $0.3 \%$ Triton X-100 in PBS), and subsequently incubated with primary antibody (rabbit anti-red fluorescent protein [anti-RFP], 1:1,000 [MBL International, catalog PM005], mouse anti-tyrosine hydroxylase [anti-TH], 1:1,000 [ImmunoStar Inc., catalog 22941] or chicken anti-GFP, 1:1,000 [Abcam, catalog ab13970]) in blocking solution overnight. The following day, the sections were washed and incubated with secondary antibody (Alexa Fluor 488/568 anti-chicken [1:1,000, catalog A11039], anti-rabbit [1:1,000, catalog A10042], or anti-mouse [1:5,000, catalog A21202], all from Invitrogen) in blocking solution for 2 hours. The sections were then washed and mounted on object glasses with VECTASHIELD HardSet Antifade Mounting Medium Reagent (Vector Laboratories). Cells expressing Drd1-tdTomato were visualized without the use of immunohistochemistry. MC4ReGFP-D1-tdTomato was analyzed in 3 mice. Sections were analyzed using a Nikon 80i microscope equipped with epifluorescence and a Zeiss Axio Observer Z1 fluorescence microscope connected to a Zeiss LSM 700 confocal unit with 405, 488, 555, and $639 \mathrm{~nm}$ diode lasers.

Dopamine receptor autoradiography. Flash-frozen brains from WT and transgenic mice were sectioned $(20 \mu \mathrm{m})$ on a Cryostat (Leica) and thaw mounted onto ethanol-washed glass slides. Slides were preincubated (15 minutes, room temperature [RT]) in washing buffer $(50 \mathrm{mM}$ Tris- $\mathrm{HCl}, \mathrm{pH}$ 7.4), then transferred to incubation buffer (120 minutes, RT; $50 \mathrm{mM}$ Tris-HCl, pH 7.4, with $120 \mathrm{mM} \mathrm{NaCl}$ and $10 \mathrm{mM} \mathrm{MgCl}$ ) containing $\left[{ }^{3} \mathrm{H}\right]$ raclopride $(4 \mathrm{nM}, 65 \mathrm{Ci} / \mathrm{mmol}$, PerkinElmer). Another set of slides containing consecutive sections was incubated in the same conditions in the presence of butaclamol $(10 \mu \mathrm{M}$, Tocris) to determine nonspecific binding. Slides were washed in ice-cold incubation buffer (15 seconds) and rinsed in ice-cold water. Then slides were dried, placed in a Hypercassette (Amersham Biosciences), and covered with a BAS-TR2025 Storage Phosphor Screen (Fujifilm). A slide containing Carbon-14 Standards (American Radiolabeled Chemicals Inc.) was exposed simultaneously for quantitative radiometric analysis.
The slides were exposed to the screen for 5 days and imaged using a phosphorimager (Typhoon FLA 7000; GE Healthcare). Images were calibrated and analyzed using Image $1.51 \mathrm{j} 8$ (NIH). Briefly, based on neuroanatomical landmarks, regions of interest (ROIs) (14 to 18 per animal) were drawn freehand along the border of the striatum and quantified by densitometry.

DREAMM and $\left[{ }^{11} \mathrm{C}\right]$ raclopride displacement PET imaging. POMCCre mice expressing hM3Dq in projection neurons from the Arc to the ventral striatum (described as part of retrograde activation in Stereotaxic surgeries) were fasted overnight. The next day, the mice received an i.p. injection of either $\mathrm{NaCl}$ or $\mathrm{CNO}(2 \mathrm{mg} / \mathrm{kg})$ and were placed in their home cages. $\mathrm{NaCl}$ or $\mathrm{CNO}$ administration occurred on 2 separate days. Approximately 15 minutes later, the mice were injected with approximately $0.5 \mathrm{mCi}$ of FDG and placed in their home cages. FDG was obtained commercially. Twenty-five minutes later, mice were anesthetized with $1.5 \%$ isoflurane, placed in a prone position on the bed of an ARGUS small animal PET/CT scanner (Sedecal), and scanned using a 20-minute static acquisition protocol. The PET data were reconstructed using the $2 \mathrm{D}$ ordered subset expectation maximization algorithm (2D-OSEM) and corrected for dead time and radioactive decay. Image processing and analysis were performed as previously described $(30,48)$.

For $\left[{ }^{11} \mathrm{C}\right]$ raclopride displacement studies, procedures were identical to those described above, except that MC4R WT and MC4R-KO mice were injected i.v. with approximately $0.3 \mathrm{mCi}(\sim 1-5 \mathrm{nmol} / \mathrm{kg})$ $\left[{ }^{11} \mathrm{C}\right]$ raclopride 20 minutes after pretreatment with either $\mathrm{NaCl}$ or LPS $(10 \mu \mathrm{g} / \mathrm{kg})$ and scanned 30 minutes later. Qualitative and quantitative assessments of PET images were performed using the PMOD software environment (PMOD Technologies). Briefly, striatal and cerebellar ROIs were delineated on an MRI mouse brain template provided with the software. Then all PET images were coregistered and spatially normalized to the combined MRI-ROI template using the Fusion module in PMOD. This led to coregistered PET-MRI images with a voxel size of $0.2 \mathrm{~mm}$ isotropic. ROI values (counts/s) were extracted from striatum and cerebellum and converted to binding ratios.

Electrophysiology. Coronal sections $(250 \mu \mathrm{m})$ containing the striatum were prepared from 11 transgenic mice expressing $\mathrm{Chr} 2$ in a Cre-dependent manner restricted to POMC neurons. Mice were deeply anesthetized with isoflurane (2.5\%) and decapitated before removal of the front brain. Slices were cut using a vibratome in ice-cold artificial cerebrospinal fluid (aCSF) containing elevated sucrose: $50 \mathrm{mM}$ sucrose, $95 \mathrm{mM} \mathrm{NaCl}, 26 \mathrm{mM} \mathrm{NaHCO}_{3}, 1.8 \mathrm{mM} \mathrm{KCl}, 1.2 \mathrm{mM} \mathrm{KH}_{2} \mathrm{PO}_{4}, 0.5 \mathrm{mM}$ $\mathrm{CaCl}_{2}, 7 \mathrm{mM} \mathrm{MgSO}_{4}$, and $15 \mathrm{mM}$ glucose. After 90 minutes, the slices were transferred to a recording chamber and perfused continuously with approximately $2 \mathrm{ml} / \mathrm{min}$ aCSF at $35^{\circ} \mathrm{C}$ containing the following: $127 \mathrm{mM} \mathrm{NaCl}, 26 \mathrm{mM} \mathrm{NaHCO}_{3}, 1.8 \mathrm{mM} \mathrm{KCl}, 1.2 \mathrm{mM} \mathrm{KH}_{2} \mathrm{PO}_{4}, 2.4 \mathrm{mM}$ $\mathrm{CaCl}_{2}, 1.3 \mathrm{mM} \mathrm{MgSO}_{4}$, and $15 \mathrm{mM}$ glucose. All solutions were oxygenated with $95 \% \mathrm{O}_{2} / 5 \% \mathrm{CO}_{2}$. Cell-attached recordings were done with an internal solution of high $\mathrm{K}^{+}$concentration: $120 \mathrm{mM} \mathrm{K}$-gluconate, 20 $\mathrm{mM} \mathrm{KCl}, 5 \mathrm{mM} \mathrm{NaCl}, 10 \mathrm{mM}$ P-creatine Tris ${ }_{2}, 10 \mathrm{mM}$ HEPES, $2 \mathrm{mM}$ Mg-ATP, and $0.3 \mathrm{mM} \mathrm{Na-GTP.} \mathrm{Recording} \mathrm{pipettes} \mathrm{(5-8} \mathrm{macrophages)}$ were pulled from borosilicate glass (World Precision Instruments). Neurons were visualized with a $\times 40$ water-immersion objective on an upright Axioskop FS microscope (Zeiss) equipped with infrareddifferential interface contrast (IR-DIC) video microscopy via an Orca-R2 CCD camera (Hamamatsu). After achieving Giga-seal, Chr2 was stimulated by flashing $470 \mathrm{~nm}$ light ( $5 \mathrm{~ms}$ pulses; $20 \mathrm{~Hz} .25 \%$ intensity) through 
the light path of the microscope using the LED powered by an LED driver (Colibri, Zeiss). A master 8-pulse generator (AMPI) controlled the timing of the light pulses. To evoke neuropeptide release, light stimulation was maintained for 1-2 minutes. Light intensity remained unchanged during the experiments, and the field of view was illuminated. Action potentials were recorded from neurons in the striatum in cell-attached mode using an EPC9 amplifier (HEKA Elektronik). Cells that increased their firing frequency by more than $40 \%$ during optic stimulation were considered responders. Out of 18 cells, 4 responded; these cells were from 4 individual animals. Out of the 4 that responded to light stimulation, 1 neuron went into whole-cell mode toward the end of the recording. For pharmacological characterization of light-evoked action potentials, $20 \mu \mathrm{M} \mathrm{DNQX}$ disodium salt (Tocris) was perfused into the bath prior to 1 minute of light stimulation, followed by a 1-minute nonlight baseline. Subsequently, HSO14 was bath applied in a final concentration of $10 \mathrm{nM}$, so that it was present during the subsequent 1-minute light stimulation. IV curves were recorded before and after each experiment to verify that the cell was still viable. Data analysis was performed offline using Igor Pro WaveMetrics.

Statistics. Results are illustrated as mean \pm SEM. All statistical analysis was based on the assumption that the samples follow a Gaussian distribution, and tests were selected accordingly. No statistical methods were used to predetermine sample sizes. Sample sizes for each experiment were chosen according to our previous or similar studies with these experimental paradigms (7). Statistical comparison of experiments with 2 groups was done using Student's 2-tailed $t$ test. When comparing more than 2 groups in an experiment with comparable variances, 1-way ANOVA or 2-way ANOVA was performed, followed by post hoc analysis with Bonferroni's multiple comparison tests to evaluate pairwise group differences. $P<0.05$ was considered statistically significant. Statistical analyses were conducted using GraphPad Prism 6 software, and voxel statistical analysis from PET-imaging results were done in MATLAB.

Study approval. All experiments involving the use of animals followed international and national guidelines and were approved by the Research Animal Care and Use Committee in Linköping, Sweden.

\section{Author contributions}

AMK, MF, and DE were responsible for the overall study design. $\mathrm{AMK}, \mathrm{MF}$, and AN performed the conditioned place preference experiments. AMK and MF conducted the operant runway, thermal place aversion, and nociceptive responding to formalin experiments. AMK and MF did stereotaxic injections of viral vectors. AMK and UK conducted the electrophysiological experiments, with supervision from BG. AMK was responsible for the histological analysis. AN performed the food-intake experiments. EM measured body temperature changes. KS and AMK executed the corticosterone experiments. MJ performed the genotyping and colony maintenance. PET scans were designed and conducted by MF and MM. JB did the autoradiography measures of dopamine-receptor binding. $\mathrm{AB}$ provided some of the mouse lines and input on the design of the study. The manuscript was written by AMK, MF, MM, and DE. All authors read and commented on the manuscript.

\section{Acknowledgments}

This study was supported by the European Research Council, the Swedish Medical Research Council, the Knut and Alice Wallenberg Foundation, the Swedish Brain foundation, the County Council of Östergötland (to DE), the National Institute on Drug Abuse Intramural Research Program (ZIA000069 to MM), and the Lars Hiertas Minne Foundation (to MF). We thank Joanna Zajdel for initial studies on the MC4R-reporter line, Haissa Brito for help in the pilot studies of LiCl-induced hypothermia, and Johan Jakobsson for input on viral vectors.

Address correspondence to: David Engblom, Department of Clinical and Experimental Medicine, Center for Social and Affective Neuroscience, House 463, Linköping University, 58185 Linköping, Sweden. Phone: 46702611302; Email: david. engblom@liu.se.
1. Johansen JP, Fields HL. Glutamatergic activation of anterior cingulate cortex produces an aversive teaching signal. Nat Neurosci. 2004;7(4):398-403.

2. Johansen JP, Fields HL, Manning BH. The affective component of pain in rodents: direct evidence for a contribution of the anterior cingulate cortex. Proc Natl Acad Sci U S A. 2001;98(14):8077-8082.

3. Craig AD. How do you feel? Interoception: the sense of the physiological condition of the body. Nat Rev Neurosci. 2002;3(8):655-666.

4. Betley JN, et al. Neurons for hunger and thirst transmit a negative-valence teaching signal. Nature. 2015;521(7551):180-185.

5. Han S, Soleiman MT, Soden ME, Zweifel LS, Palmiter RD. Elucidating an affective pain circuit that creates a threat memory. Cell. 2015;162(2):363-374.

6. Singh AK, et al. Prostaglandin-mediated inhibition of serotonin signaling controls the affective component of inflammatory pain. J Clin Invest. 2017;127(4):1370-1374.

7. Fritz M, et al. Prostaglandin-dependent modulation of dopaminergic neurotransmission elicits inflammation-induced aversion in mice. J Clin
Invest. 2016;126(2):695-705.

8. Liu C, Lee S, Elmquist JK. Circuits controlling energy balance and mood: inherently intertwined or just complicated intersections? Cell Metab. 2014;19(6):902-909.

9. Luquet S, Perez FA, Hnasko TS, Palmiter RD. NPY/AgRP neurons are essential for feeding in adult mice but can be ablated in neonates. $\mathrm{Sci}$ ence. 2005;310(5748):683-685.

10. Carter ME, Soden ME, Zweifel LS, Palmiter RD. Genetic identification of a neural circuit that suppresses appetite. Nature. 2013;503(7474):111-114.

11. Gropp E, et al. Agouti-related peptide-expressing neurons are mandatory for feeding. Nat Neurosci. 2005;8(10):1289-1291.

12. Krashes MJ, Lowell BB, Garfield AS. Melanocortin-4 receptor-regulated energy homeostasis. Nat Neurosci. 2016;19(2):206-219.

13. Balthasar N, et al. Divergence of melanocortin pathways in the control of food intake and energy expenditure. Cell. 2005;123(3):493-505.

14. Lim BK, Huang KW, Grueter BA, Rothwell PE, Malenka RC. Anhedonia requires MC4Rmediated synaptic adaptations in nucleus accumbens. Nature. 2012;487(7406):183-189.
15. Rossi J, et al. Melanocortin-4 receptors expressed by cholinergic neurons regulate energy balance and glucose homeostasis. Cell Metab. 2011;13(2):195-204.

16. Ngampramuan S, et al. Thermoregulatory correlates of nausea in rats and musk shrews. Oncotarget. 2014;5(6):1565-1575.

17. Lammel S, et al. Input-specific control of reward and aversion in the ventral tegmental area. Nature. 2012;491(7423):212-217.

18. Soares-Cunha C, Coimbra B, Sousa N, Rodrigues AJ. Reappraising striatal D1- and D2-neurons in reward and aversion. Neurosci Biobehav Rev. 2016;68:370-386.

19. Pignatelli M, Bonci A. Role of dopamine neurons in reward and aversion: a synaptic plasticity perspective. Neuron. 2015;86(5):1145-1157.

20. Schultz W, Dayan P, Montague PR. A neural substrate of prediction and reward. Science. 1997;275(5306):1593-1599.

21. Schultz W. Dopamine reward prediction-error signalling: a two-component response. Nat Rev Neurosci. 2016;17(3):183-195.

22. Morris ED, Yoder KK. Positron emission tomography displacement sensitivity: predicting binding 
potential change for positron emission tomography tracers based on their kinetic characteristics. JCereb Blood Flow Metab. 2007;27(3):606-617.

23. Castro DC, Berridge KC. Opioid hedonic hotspot in nucleus accumbens shell: $\mu, \delta$, and $\kappa$ maps for enhancement of sweetness "liking" and "wanting". J Neurosci. 2014;34(12):4239-4250.

24. Tan KR, et al. GABA neurons of the VTA drive conditioned place aversion. Neuron. 2012;73(6):1173-1183.

25. Roth BL. DREADDs for neuroscientists. Neuron. 2016;89(4):683-694.

26. Kishi T, Aschkenasi CJ, Lee CE, Mountjoy KG, Saper CB, Elmquist JK. Expression of melanocortin 4 receptor mRNA in the central nervous system of the rat. J Comp Neurol. 2003;457(3):213-235.

27. Cui H, Mason BL, Lee C, Nishi A, Elmquist JK, Lutter M. Melanocortin 4 receptor signaling in dopamine 1 receptor neurons is required for procedural memory learning. Physiol Behav. 2012;106(2):201-210.

28. Gomez JL, et al. Chemogenetics revealed: DREADD occupancy and activation via converted clozapine. Science. 2017;357(6350):503-507.

29. Ghamari-Langroudi M, et al. G-proteinindependent coupling of MC4R to Kir7.1 in hypothalamic neurons. Nature. 2015;520(7545):94-98.

30. Michaelides M, et al. Whole-brain circuit dissection in free-moving animals reveals cellspecific mesocorticolimbic networks. JClin Invest. 2013;123(12):5342-5350.

31. Contreras M, Ceric F, Torrealba F. Inactivation of the interoceptive insula disrupts drug craving and malaise induced by lithium. Science.
2007;318(5850):655-658.

32. Cui H, Lutter M. The expression of MC4Rs in D1R neurons regulates food intake and locomotor sensitization to cocaine. Genes Brain Behav. 2013;12(6):658-665.

33. Marks DL, Ling N, Cone RD. Role of the central melanocortin system in cachexia. Cancer Res. 2001;61(4):1432-1438.

34. Stuber GD, et al. Excitatory transmission from the amygdala to nucleus accumbens facilitates reward seeking. Nature. 2011;475(7356):377-380

35. Britt JP, Benaliouad F, McDevitt RA, Stuber GD, Wise RA, Bonci A. Synaptic and behavioral profile of multiple glutamatergic inputs to the nucleus accumbens. Neuron. 2012;76(4):790-803.

36. Qi J, Zhang S, Wang HL, Barker DJ, MirandaBarrientos J, Morales M. VTA glutamatergic inputs to nucleus accumbens drive aversion by acting on GABAergic interneurons. Nat Neurosci. 2016;19(5):725-733.

37. Zhu Y, Wienecke CF, Nachtrab G, Chen X. A thalamic input to the nucleus accumbens mediates opiate dependence. Nature. 2016;530(7589):219-222.

38. Delfs JM, Zhu Y, Druhan JP, Aston-Jones G. Noradrenaline in the ventral forebrain is critical for opiate withdrawal-induced aversion. Nature. 2000;403(6768):430-434.

39. Bruchas MR, et al. Selective $\mathrm{p} 38 \alpha$ MAPK deletion in serotonergic neurons produces stress resilience in models of depression and addiction. Neuron. 2011;71(3):498-511.

40. Land BB, et al. Activation of the kappa opioid receptor in the dorsal raphe nucleus mediates the aversive effects of stress and reinstates drug seeking. Proc Natl Acad Sci U S A. 2009;106(45):19168-19173.

41. Balthasar N, et al. Leptin receptor signaling in POMC neurons is required for normal body weight homeostasis. Neuron. 2004;42(6):983-991.

42. Parkitna JR, et al. Loss of the serum response factor in the dopamine system leads to hyperactivity. FASEB J. 2010;24(7):2427-2435.

43. Engblom D, et al. Glutamate receptors on dopamine neurons control the persistence of cocaine seeking. Neuron. 2008;59(3):497-508.

44. Madisen L, et al. A toolbox of Cre-dependent optogenetic transgenic mice for light-induced activation and silencing. Nat Neurosci. 2012;15(5):793-802.

45. Xiao C, et al. Brain transit and ameliorative effects of intranasally delivered anti-amyloid- $\beta$ oligomer antibody in 5XFAD mice. J Alzheimers Dis. 2013;35(4):777-788.

46. Bardo MT, Rowlett JK, Harris MJ. Conditioned place preference using opiate and stimulant drugs: a meta-analysis. Neurosci Biobehav Rev. 1995;19(1):39-51.

47. Geist TD, Ettenberg A. A simple method for studying intravenous drug reinforcement in a runaway. Pharmacol Biochem Behav. 1990;36(3):703-706.

48. Michaelides M, Hurd YL. DREAMM: a biobehavioral imaging methodology for dynamic in vivo whole-brain mapping of cell type-specific functional networks. Neuropsychopharmacology. 2015;40(1):239-240. 